\title{
Endometriosis of Abdominal Wall Scarring
}

\author{
Abdelmounim Elkhamri", Abdellatif Settaf, Hamid Mohammadine
}

Surgical Department B, Faculty of Medicine and Pharmacy, Mohammed 5 University, Ibn Sina Hospital, Rabat, Morocco

*Corresponding author: Abdelmounim Elkhamri

Abstract

Endometriosis of the wall is a very rare pathology. Its pathophysiology remains unclear. It occurs on scars from gynaecological or obstetrical surgery. We report the case of a patient presenting a cyclic pain, at the level of the caesarean section scar, at the clinical examination a mass of $7 \mathrm{cmx} 5 \mathrm{~cm}$ localized at the level of the scar of the eventration cure. Pelvic computed tomography (CT) scan established the presence of a hypodense formation, measuring 75/55 mm extended over $40 \mathrm{~mm}$, which rises in its periphery after injection of contrast agent, opposite the operative scar of the eventration cure at the expense of the skin tissue. The treatment was surgical with complete removal of the lesion and a pathological study was performed which confirmed parietal endometriosis. The postoperative follow-up was simple. Through our case, we draw the practitioners' attention to the great interest of evoking the diagnosis of parietal endometriosis each time a patient presents a mass of the wall with cyclic pain, in the close or distant aftermath of gynaecological surgery. This should systematically lead to a wide excision, followed by parietal reconstruction using prosthetic reinforcement if necessary, because of the risk of cancerization, the prognosis for which is considered to be poor.

Keywords: Endometriosis, cancerization, computed tomography (CT), hypodense formation.

Copyright @ 2020: This is an open-access article distributed under the terms of the Creative Commons Attribution license which permits unrestricted use, distribution, and reproduction in any medium for non-commercial use (NonCommercial, or CC-BY-NC) provided the original author and source are credited.

\section{DEFINITION}

Endometriosis is characterised by the formation of tissue outside the uterus formed by endometrial cells. Thus, endometrium starts to form elsewhere in the body [1].

Its location on the abdominal wall is rare. Parietal endometriosis accounts for $1-2 \%$ of extra genital endometriosis cases [2].

The endometriosis of the abdominal wall can be located in different places: the rectus abdominis, the umbilicus, caesarean section scars, the skin, the adjacent tissues of abdominal or pelvic surgery scars, the path of an amniocentesis needle, and on the laparoscopic trocar port.

\section{Medical Observations}

A 46-year-old woman was hospitalized for surgical management of pelvic swelling. The mother of
3 living children and carrier of a tri-scarred uterus (three Caesarean sections), the last Caesarean section was 16 years ago. This patient has a surgical history of a platelet rupture 15 years ago. She consulted for pain at the level of the scar of the eventration cure, this cyclic, catamenial pain at the level of the scar of the eventration cure for a year. The clinical examination found a nodular mass in the middle of the eventration scar of about 75/55 mm, which was dark in colour and painful on palpation (Figure-1). Gynaecological examination and pelvic touching were unremarkable. Pelvic computed tomography (CT) scan established the presence of a hypodense formation, measuring $75 / 55 \mathrm{~mm}$, extending over $40 \mathrm{~mm}$, and rising around the periphery of the operative scar at the expense of the skin tissue after injection of contrast medium (Figure2 ). The procedure performed was total surgical removal of the parietal nodular mass. The anatomicopathological study was in favour of parietal endometriosis (Figure3).The postoperative follow-up was simple. 


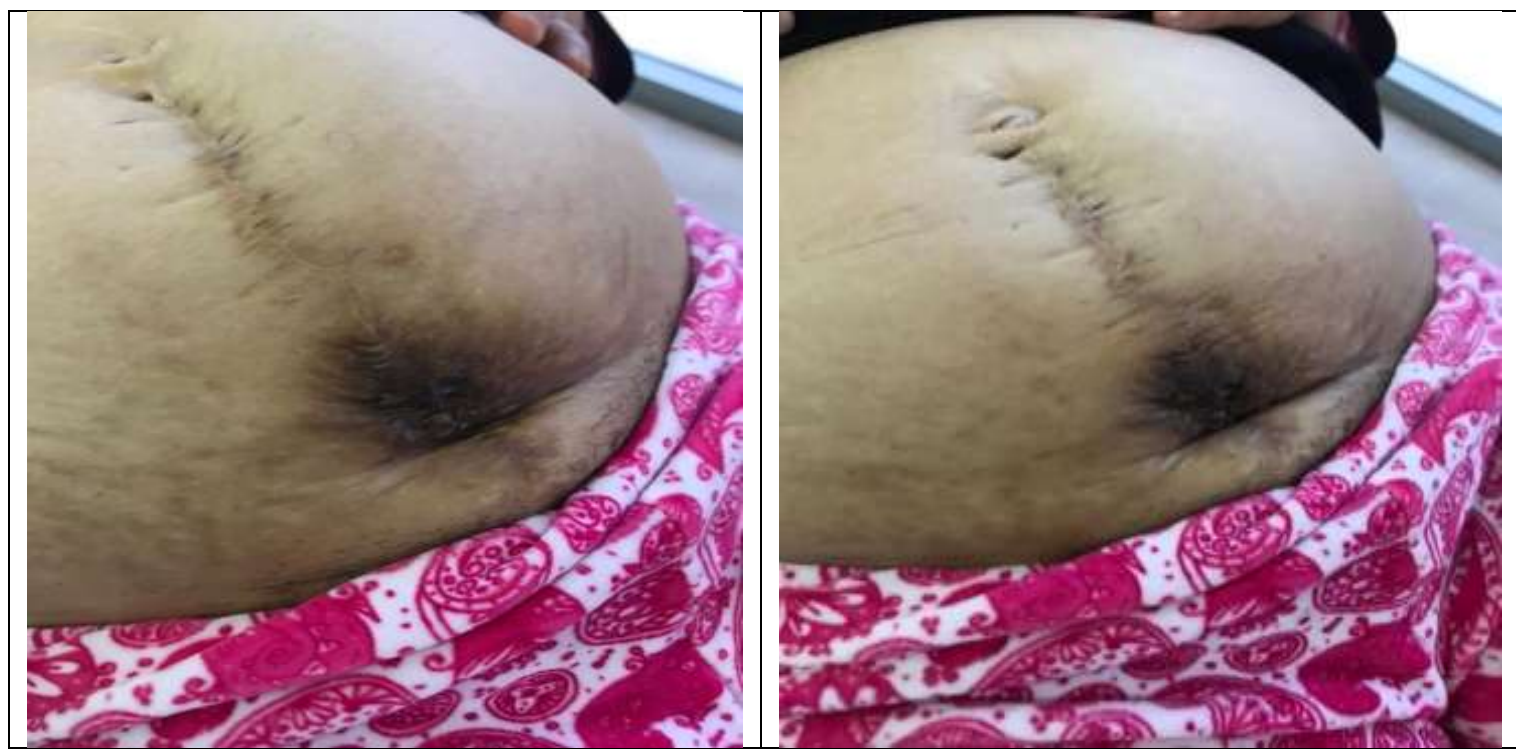

Fig-1
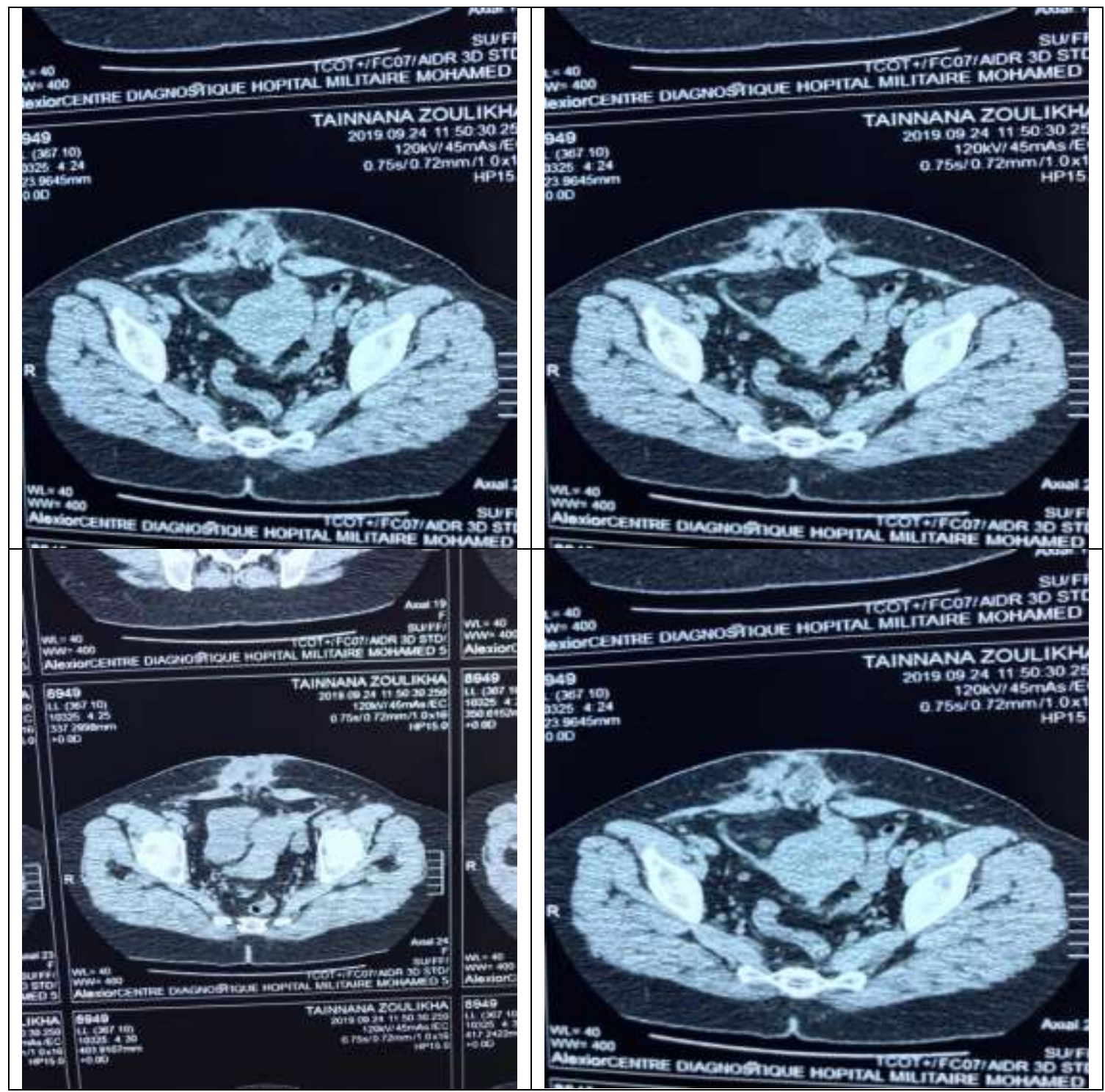

Fig-2 


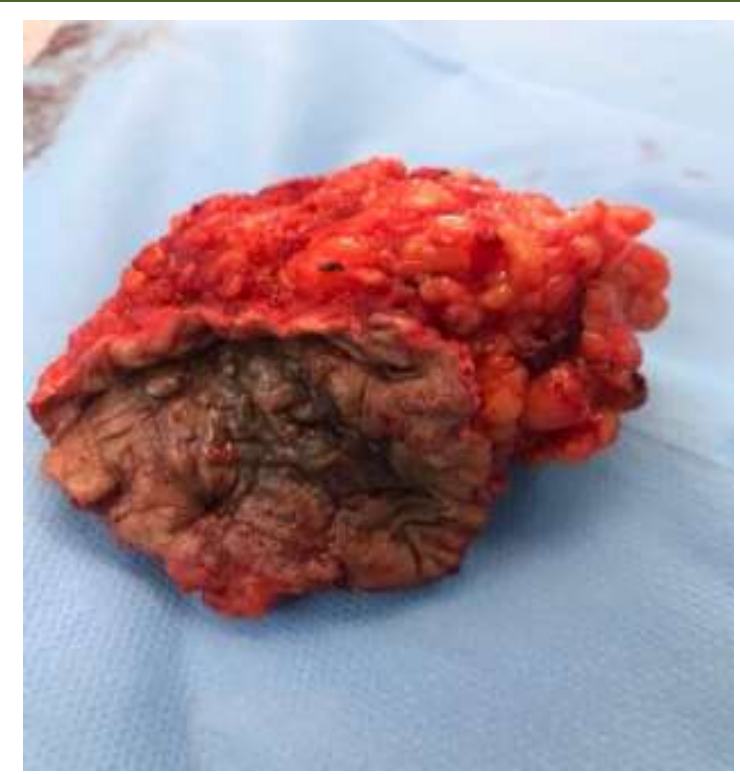

Fig-3

\section{DiscuSSION}

Endometriosis of the abdominal wall is a pathology whose initial diagnosis is not always easy [1]. It represents 0.03 to $2 \%$ of extra genital endometriosis [3-5].

Its pathophysiological mechanism is explained by an endometrial cell transplant during surgery favored by estrogens producing endometriomas [6].

Clinically it manifests as: a palpable mass opposite a scar gradually increasing in volume [3]; cyclic pain which is an important element of orientation but does not confirm the diagnosis [3, 7, 8]. Palpation of the lesion should allow its size and location to be appreciated in depth; the lesion frequently invades the abdominal muscles and their sheath. In $37 \%$ of cases, the diagnosis is pathological discovery $[9,10]$.

Ultrasound is the first examination on request, it is an easy examination which allows diagnostic orientation and preoperative assessment, even if it does not confirm a formal diagnosis. It confirms the typically intramuscular parietal origin of the mass suspected on clinical examination. It specifies the size, the contours and its relationships with the adjacent structures [3]. Our patient did not benefit from an ultrasound.

CT and magnetic resonance imaging (MRI) can be used to diagnose parietal endometriosis [11]. The diagnosis was made preoperatively by computed tomography in our patient by showing an iso-dense tissue lesion process, anterior and medial parietal, infiltrating the subcutaneous fat and the underlying muscles [11]. MRI constitutes the examination of choice to confirm the diagnosis in case of doubt because it allows highlighting the iron content of hemosiderin deposits in endometriomas. MRI remains more sensitive than CT for detecting small lesions [12]. MRI was not performed in our patient.

Parietal endometriosis is suspected clinically and its diagnosis is confirmed by anatomicopathological examination of the lesion [4-7]. The examination revealed endometrial glands of variable sizes often of cystic type associated with a cytogenic chorion and a lymphocytic inflammation.

The ectopic situation of these endometrial glands therefore corresponds to the diagnosis of external endometriosis

The treatment of these lesions is based on surgical excision. This excision must be completed in order to remove the entire lesion, the risk of recurrence exists in the event of incomplete excision. This complete surgery can be decaying requiring a parietal reconstruction with parietoplasty [13].

Malignant transformation of endometriosis is rare. Several authors have found a statistically significant association between the use of estrogens and the malignant transformation of endometriosis medical treatment (LHRH agonists or progestins) makes it possible to improve the symptomatology by reducing the painful and inflammatory nature of lesions of endometriosis of parietal scar, this cannot bring about a cure and the lesions recur quickly when stopping these therapies [3]. This medical treatment can be done postoperatively, our patient did not benefit from medical treatment (LHRH agonists or progestins).

$\mathrm{CO} 2$ laser photocoagulation has also been reported to be effective but remains uncommon [7]. Recurrences are not uncommon, up to 10 to $15 \%$. The recurrence rate is correlated with the size and depth of the lesion [3]. Prevention in case of laparotomy is based on the abundant washing of the abdominal cavity and the scar at the end of the intervention as well as the change of gloves for the time of parietal closure, while in laparoscopy, the extraction of the operating pieces in a protective bag and abundant washing of the pelvic cavity should be systematic. Thus, these measures are part of good surgical practice although their benefit has never been demonstrated [3]

\section{CONCLUSION}

We draw the practitioners' attention to the great interest of evoking the diagnosis of parietal endometriosis each time a patient presents a mass of the wall with cyclic pain, in the near or distant consequences of gynecological surgery. This should systematically lead to a wide excision, followed by a parietal reconstruction using prosthetic reinforcement if necessary, because of the risk of cancerization, the prognosis of which is considered to be poor. 


\section{REFERENCES}

1. Sinha R, Kumar M, Matah M. Abdominal scar endometriosis after Cesarean section: a rare entity. Australas Med J. 2011; 4(1):60-2.

2. Lamblin G, Mathevet P, Buenerd A. Endométriose pariétale sur cicatrice abdominale: à propos de trois observations. J Gynecol Obstet Biol Reprod (Paris). 1999 Jun; 28(3): 271-4.

3. Audebert A. Les endométrioses iatrogènes de la femme avant la ménopause: principaux enjeux. Gynécologie Obstétrique \& Fertilité. 2013; 41:322-327.

4. Hafidi R, Kouach J, Yakka M, Salek G, Oukabli M, Moussaoui RD, Dehayni M. L?endométriose cicatricielle de la paroi abdominale. Imagerie de la Femme. 2011; 21(3): 125-127.

5. Victory R, Diamond MP, Johns DA. Villar's nodule: a case report and systematic literature review of endometriosis externa of the umbilicus. J Minim Invasive Gynecol. 2007 JanFeb;14(1):2332.

6. Thylan S. Re: abdominal wall endometrioma in a laparoscopic trocar tract: a case report. Am Surg. 1996 Jul; 62(7): 617.

7. Jayi S, Bouguern H, Bouchikhi C, Amhager N, Chaara H, Melhouf MA, Banani A. Greffe endométriosique postobstétricale, à propos de 2 cas. Médecine du Maghreb. 2008; 154: 21-25.

8. Khlifi A, Kouira M, Anis M, Sonia Z, Ines L, Habib S, Mohamed B, Samir H, Hedi K. Endométriose pariétale sur cicatrice de césarienne: à propos de 3 cas. Imagerie de la Femme. 2012 Sep 1;22(3):156-61.

9. Jaouad K, Abdellah BH, Mehdi E, Driss M, Mohamed D. L'endométriose ombilicale: à propos d'un cas. Médecine de la Reproduction. 2010 Oct 1;12(4):324-7.

10. Picod G, Boulanger L, Bounoua F, Leduc F, Duval G. Endométriose pariétale sur cicatrice de césarienne: à propos de 15 cas. Gynécologie obstétrique \& fértilité. 2006 Jan 1;34(1):8-13.

11. Merran S, Karila-Cohen P. Endométriose sous cutanée sur cicatrice de la paroi abdominale antérieure: à propos de deux observations. J Radiol. 2004 Apr; 85(4 Pt 1): 409-10.

12. Simsir A, Thorner K, Waisman J, Cangiarella J. Endometriosis in abdominal scars: a report of three cases diagnosed by fineneedle aspiration biopsy. Am Surg. 2001 Oct; 67(10): 984-6.

13. Emre A, Akbulut S, Yilmaz M, Bozdag Z. Laparoscopic trocar Port site endometriosis: a case report and brief literature review. Int Surg. 2012 Apr-Jun; 97(2): 135-9. 